\title{
PID Control of Second-Order Systems with Hysteresis*
}

\author{
Bayu Jayawardhana, Hartmut Logemann ${ }^{\dagger} \&$ Eugene P Ryan \\ Department of Mathematical Sciences \\ University of Bath \\ Bath BA2 7AY, UK
}

May 2007

Revised: October 2007

\begin{abstract}
The efficacy of proportional, integral and derivative (PID) control for set point regulation and disturbance rejection is investigated in a context of secondorder systems with hysteretic components. Two basic structures are studied: in the first, the hysteretic component resides (internally) in the restoring force action of the system ("hysteretic spring" effects); in the second, the hysteretic component resides (externally) in the input channel (e.g. piezo-electric actuators). In each case, robust conditions on the PID gains, explicitly formulated in terms of the system data, are determined under which asymptotic tracking of constant reference signals and rejection of constant disturbance signals is guaranteed.
\end{abstract}

Keywords. Hysteresis, Nonlinear systems, PID control, Tuning regulators.

\section{Introduction}

With reference to Figures 1 and 2, we consider PID control of single-input (mechanical) systems of the following two forms:

$$
\begin{gathered}
m \ddot{x}+c \dot{x}+\Phi(x)=u+d, \\
m \ddot{x}+c \dot{x}+k x=\Phi\left(u+d_{2}\right)+d_{1},
\end{gathered}
$$

with input $t \mapsto u(t) \in \mathbb{R}$ and constant (but unknown) disturbances $d, d_{1}, d_{2} \in \mathbb{R}$. In a mechanical context, $x(t)$ represents displacement at time $t \in \mathbb{R}_{+}:=[0, \infty)$, $m$ and $c$ are the mass and the damping constant, and, in (2), $k$ is a linear spring constant. In the case of (1), the operator $\Phi$ models a restoring force which may 


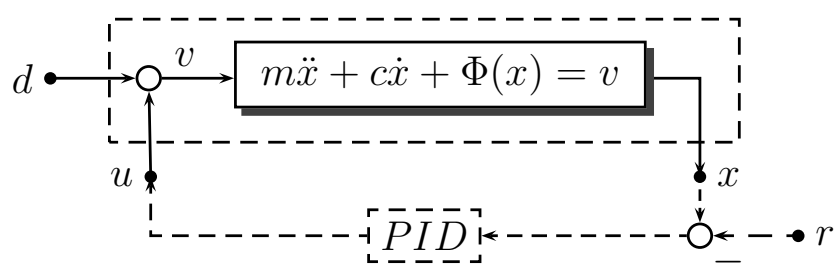

Figure 1: System of form (1)

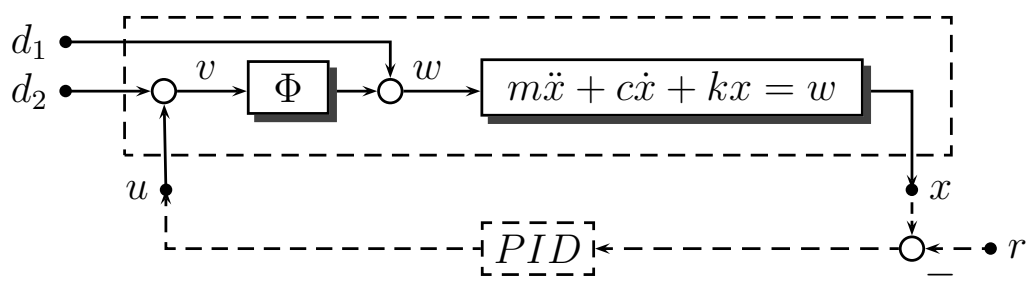

Figure 2: System of form (2)

exhibit hysteresis phenomena, a particular example of which is the "hysteric spring" model discussed in, for example, $[2,18]$. In the case of $(2)$, the operator $\Phi$ models hysteretic actuation. Such hysteretic effects arise in, for example, micro-positioning control problems using piezo-electric actuators or smart actuators, as investigated in, inter alia, $[1,4,5,6,9,10,16,17,19]$.

Motivated by a recent study in [7], for each of the above system structures we will investigate the efficacy of a PID controller of the form

$$
u(t)=-k_{p}(x(t)-r)-k_{d} \dot{x}(t)-k_{i} \int_{0}^{t}(x(\tau)-r) \mathrm{d} \tau+u_{0},
$$

where $u_{0}$ is the initial condition on the integrator, $k_{p}, k_{i}, k_{d} \geq 0$ are the controller gains and $r$ is a constant reference signal to be tracked. The latter is a distinguishing feature of the present paper vis à vis [7] (wherein constant non-zero reference signals cannot be handled). Moreover, the investigation in [7] is concerned with systems of form (1) and is focussed on one particular hysteresis component, namely the Bouc-Wen model $[8,20]$. By contrast, in this paper we deal with a large class of rate-independent causal hysteresis operators which includes the play operator, stop operator, backlash operator and Preisach operators. These operators are briefly discussed in Section 2, a more detailed discussion can be found in [3, 11, 14].

The rapidly expanding literature (of which [1] - [20] is but a representative sample) on analysis and control of systems with hysteresis attests to the growing importance of the field to both control theorists and practitioners. The analytical framework of the present paper is based on frequency-domain conditions developed recently

*This work was supported by the UK Engineering \& Physical Sciences Research Council under Grant GR/S94582/01

${ }^{\dagger}$ Corresponding author, e-mail: hl@maths.bath.ac.uk 
in $[12,13]$ which ensure existence, regularity and certain asymptotic properties of solutions of a feedback interconnection of a linear (possibly infinite dimensional) system and a hysteresis operator $\Phi$. Within this framework, the main contribution of the paper is as follows. For each of the two underlying system structures (1) and (2), robust design criteria - formulated explicitly in terms of bounds on the plant parameters $m, c, k$ and on a Lipschitz-type constant $\lambda$ associated with the hysteresis operator $\Phi$ - are developed under which disturbance rejection is assured and the tracking error $x(t)-r$ converges, as $t \rightarrow \infty$, to zero at exponential rate. We emphasize the robustness aspect: the latter performance is achieved by PID control for which computation of the associated PID gains requires only the availability of bounds on the parameters $m, c, k$ and $\lambda$; in particular (and in contrast to [7]), precise knowledge of the hysteresis operator $\Phi$ is not required (see, in particular, Remark 4.2 and Remark 4.4 below).

Notation. We conclude this introduction with some remarks on terminology and notation. As usual, we denote the space of continuous functions $I \rightarrow \mathbb{R}, I \subset \mathbb{R}$ an interval, by $C(I)$. A function $f \in C\left(\mathbb{R}_{+}\right)$is said to be piecewise monotone if, for some strictly increasing unbounded sequence $\left(t_{i}\right)_{i=0}^{\infty}$ in $\mathbb{R}_{+}$with $t_{0}=0, f$ is monotone on $\left[t_{i-1}, t_{i}\right]$ for all $i \in \mathbb{N}$ : the space of all such piecewise monotone functions is denoted by $C_{\mathrm{pm}}\left(\mathbb{R}_{+}\right)$. The (Banach) space of measurable functions $f: \mathbb{R}_{+} \rightarrow \mathbb{R}$ such that $\|f\|_{L^{p}}:=\int_{0}^{\infty}|f(t)|^{p} \mathrm{~d} t<\infty, 1 \leq p<\infty$, is denoted by $L^{p}\left(\mathbb{R}_{+}\right)$. For $f \in L^{p}\left(\mathbb{R}_{+}\right)$ and $T>0, f_{T}$ denotes the concatenation of the functions $\left.f\right|_{[0, T]}$ and 0 , given by

$$
f_{T}(t):= \begin{cases}f(t), & t \in[0, T] \\ 0, & t \in(T, \infty) .\end{cases}
$$

The space $L_{\text {loc }}^{p}\left(\mathbb{R}_{+}\right)$consists of all measurable functions $f: \mathbb{R}_{+} \rightarrow \mathbb{R}$ such that $f_{T} \in L^{p}\left(\mathbb{R}_{+}\right)$for all $T>0$. By $W_{\text {loc }}^{1,1}\left(\mathbb{R}_{+}\right)$we denote the space of locally absolutely continuous real-valued functions defined on $\mathbb{R}_{+}$, that is, $f \in W_{\text {loc }}^{1,1}\left(\mathbb{R}_{+}\right)$if and only if there exists $g \in L_{\text {loc }}^{1}\left(\mathbb{R}_{+}\right)$such that $f(t)=f(0)+\int_{0}^{t} g(s) \mathrm{d} s$ for all $t \in \mathbb{R}_{+}$.

Let $\alpha \in \mathbb{R}$. The $\alpha$-exponentially weighted $L^{p}$-space of functions $\mathbb{R}_{+} \rightarrow \mathbb{R}$ is defined as

$$
L_{\alpha}^{p}\left(\mathbb{R}_{+}\right):=\left\{f: f(\cdot) \exp (-\alpha \cdot) \in L^{p}\left(\mathbb{R}_{+}\right)\right\}
$$

which, endowed with the norm

$$
\|f\|_{L_{\alpha}^{p}\left(\mathbb{R}_{+}\right)}=\|f(\cdot) \exp (-\alpha \cdot)\|_{L^{p}\left(\mathbb{R}_{+}\right)}=\left(\int_{0}^{\infty}\left|e^{-\alpha t} f(t)\right|^{p} d t\right)^{1 / p},
$$

is a Banach space.

A function $u \in C\left(\mathbb{R}_{+}\right)$is ultimately non-decreasing (non-increasing) if there exists $\tau \in \mathbb{R}_{+}$such that $u$ is non-decreasing (non-increasing) on $[\tau, \infty) ; u$ is said to be approximately ultimately non-decreasing (non-increasing), if for all $\varepsilon>0$, there exists an ultimately non-decreasing (non-increasing) function $v \in C\left(\mathbb{R}_{+}\right)$such that

$$
|u(t)-v(t)| \leq \varepsilon, \quad \forall t \in \mathbb{R}_{+} .
$$

The unit step function is denoted by $\theta$ and $\star$ denotes convolution. Finally, we set $\mathbb{R}^{*}:=\mathbb{R} \backslash\{0\}$. 


\section{Hysteresis operators}

An operator $\Psi: C\left(\mathbb{R}_{+}\right) \rightarrow C\left(\mathbb{R}_{+}\right)$is said to be causal if, for all $\tau \geq 0$ and all $v_{1}, v_{2} \in C\left(\mathbb{R}_{+}\right), v_{1}=v_{2}$ on $[0, \tau]$ implies that $\Psi\left(v_{1}\right)=\Psi\left(v_{2}\right)$ on $[0, \tau]$. The following remark shows that causal operators can be extended to "localized" versions of the domain space.

Remark 2.1. Let $I=[0, T]$, where $0<T<\infty$, or $I=[0, T)$, where $0<T \leq \infty$. For $\tau \in I$, define $Q_{\tau}: C(I) \rightarrow C\left(\mathbb{R}_{+}\right)$

$$
\left(Q_{\tau} u\right)(t)= \begin{cases}u(t), & 0 \leq t \leq \tau \\ u(\tau), & t>\tau\end{cases}
$$

If $\Psi: C\left(\mathbb{R}_{+}\right) \rightarrow C\left(\mathbb{R}_{+}\right)$is causal, then $\Psi$ extends in a natural way to an operator $C(I) \rightarrow C(I)$ : for $v \in C(I)$ simply set

$$
(\Psi(v))(t):=\left(\Psi\left(Q_{\tau} v\right)\right)(t), \quad 0 \leq t \leq \tau, \tau \in I .
$$

Causality of $\Psi$ guarantees that this definition does not depend on the choice of $\tau$ and so $\Psi(v)$ is a well-defined function on $C(I)$ for any $v \in C(I)$.

We will not distinguish notationally between the original causal operator and its localization.

A function $f: \mathbb{R}_{+} \rightarrow \mathbb{R}_{+}$is a time transformation if $f$ is continuous and nondecreasing and $\lim _{t \rightarrow \infty} f(t)=\infty$. An operator $\Phi: C\left(\mathbb{R}_{+}\right) \rightarrow C\left(\mathbb{R}_{+}\right)$is rate independent if, for every time transformation $f$,

$$
(\Phi(u \circ f))(t)=(\Phi(u))(f(t)), \quad \forall u \in C\left(\mathbb{R}_{+}\right), \quad \forall t \in \mathbb{R}_{+} .
$$

The operator $\Phi: C\left(\mathbb{R}_{+}\right) \rightarrow C\left(\mathbb{R}_{+}\right)$is said to be a hysteresis operator if $\Phi$ is causal and rate independent.

The numerical value set, NVS $\Phi$, of a hysteresis operator $\Phi$ is defined by

$$
\operatorname{NVS} \Phi:=\left\{(\Phi(u))(t): u \in C\left(\mathbb{R}_{+}\right), t \in \mathbb{R}_{+}\right\}
$$

For $w \in C([0, \alpha])($ with $\alpha \geq 0)$ and $\gamma, \delta>0$, we define

$$
\mathcal{C}(w ; \delta, \gamma):=\left\{v \in C([0, \alpha+\gamma]):\left.v\right|_{[0, \alpha]}=w, \max _{t \in[\alpha, \alpha+\gamma]}|v(t)-w(\alpha)| \leq \delta\right\} .
$$

We will have occasion to impose some or all of the following conditions on the hysteresis operator $\Phi: C\left(\mathbb{R}_{+}\right) \rightarrow C\left(\mathbb{R}_{+}\right)$:

(N1) If $u \in W_{\text {loc }}^{1,1}\left(\mathbb{R}_{+}\right)$, then $\Phi(u) \in W_{\text {loc }}^{1,1}\left(\mathbb{R}_{+}\right)$;

(N2) The operator $\Phi$ is monotone in the sense that, if $u \in W_{\text {loc }}^{1,1}\left(\mathbb{R}_{+}\right)$, then

$$
(\Phi(u))^{\prime}(t) u^{\prime}(t) \geq 0, \quad \text { a.e. } t \in \mathbb{R}_{+} ;
$$


(N3) There exists $\lambda>0$ such that for all $\alpha \geq 0$ and $w \in C([0, \alpha])$, there exist constants $\gamma, \delta>0$ such that

$$
\max _{\tau \in[\alpha, \alpha+\gamma]}|(\Phi(u))(\tau)-(\Phi(v))(\tau)| \leq \lambda \max _{\tau \in[\alpha, \alpha+\gamma]}|u(\tau)-v(\tau)|, \quad \forall u, v \in \mathcal{C}(w ; \delta, \gamma)
$$

(N4) For all $\alpha \in \mathbb{R}_{+}$and all $u \in C([0, \alpha))$, there exist $c>0$ such that

$$
\max _{\tau \in[0, t]}|(\Phi(u))(\tau)| \leq c\left(1+\max _{\tau \in[0, t]}|u(\tau)|\right), \quad \forall t \in[0, \alpha)
$$

(N5) If $u \in C\left(\mathbb{R}_{+}\right)$is approximately ultimately non-decreasing and $\lim _{t \rightarrow \infty} u(t)=$ $\infty$, then $(\Phi(u))(t)$ and $(\Phi(-u))(t)$ converge, as $t \rightarrow \infty$, to sup NVS $\Phi$ and inf NVS $\Phi$, respectively;

(N6) If, for $u \in C\left(\mathbb{R}_{+}\right), \lim _{t \rightarrow \infty}(\Phi(u))(t) \in \operatorname{int} \operatorname{NVS} \Phi$, then $u$ is bounded.

These technical assumptions are invoked in Theorems 3.1 and 3.2 below (which underpin the results of the paper): moreover, they are natural in the sense that they hold for the most commonly encountered hysteresis operators: relay, elasticplastic, backlash, Prandtl, Preisach.

We mention that, if a hysteresis operator $\Phi$ satisfies (N5), then NVS $\Phi$ is an interval. Furthermore, we remark that many hysteresis operators (see, for example, [3, 11]) are Lipschitz continuous in the sense that

$$
\sup _{\tau \in \mathbb{R}_{+}}|(\Phi(u))(\tau)-(\Phi(v))(\tau)| \leq \lambda \sup _{\tau \in \mathbb{R}_{+}}|u(\tau)-v(\tau)|, \quad \forall u, v \in C\left(\mathbb{R}_{+}\right),
$$

for some $\lambda>0$, in which case (N3) and (N4) are (trivially) satisfied and, furthermore, (N1) holds (see [11]).

In the following, we briefly describe the backlash, elastic-plastic and Preisach operators which are widely adopted as hysteresis models in engineering applications.

Backlash operator. The backlash (or play) operator, widely used in mechanical models (of, for example, gear trains or of hydraulic servovalves), has been discussed rigorously in many references, see for example $[3,11,14]$. With a view to giving a precise definition of backlash, we first define, for each $h \in \mathbb{R}_{+}$, the function $b_{h}: \mathbb{R}^{2} \rightarrow \mathbb{R}$ by

$$
b_{h}(v, w):=\max \{v-h, \min \{v+h, w\}\} .
$$

For all $h \in \mathbb{R}_{+}$and all $\xi \in \mathbb{R}$, we introduce an operator $\mathcal{B}_{h, \xi}$ defined on the space $C_{\mathrm{pm}}\left(\mathbb{R}_{+}\right)$of piecewise monotone functions, by defining, for every $u \in C_{\mathrm{pm}}\left(\mathbb{R}_{+}\right)$,

$$
\left(\mathcal{B}_{h, \xi}(u)\right)(t)= \begin{cases}b_{h}(u(0), \xi) & \text { for } t=0 \\ b_{h}\left(u(t),\left(\mathcal{B}_{h, \xi}(u)\right)\left(t_{i}\right)\right) & \text { for } t_{i-1}<t \leq t_{i}, \quad i \in \mathbb{N}\end{cases}
$$

where $0=t_{0}<t_{1}<t_{2}<\ldots$ is a partition of $\mathbb{R}_{+}$, such that $u$ is monotone on each of the intervals $\left[t_{i-1}, t_{i}\right], i \in \mathbb{N}$. Here $\xi$ plays the role of an "initial state". It is well 
known, see, for example, [3, page 42], that the operator $\mathcal{B}_{h, \xi}: C_{\mathrm{pm}}\left(\mathbb{R}_{+}\right) \rightarrow C\left(\mathbb{R}_{+}\right)$ can be extended uniquely to a hysteresis operator $\mathcal{B}_{h, \xi}: C\left(\mathbb{R}_{+}\right) \rightarrow C\left(\mathbb{R}_{+}\right)$; moreover, the extended operator is Lipschitz continuous (in the sense of (5)) with Lipschitz constant $\lambda=1$ and satisfies (N1)-(N6) (see, for example, [11, Proposition 5.4]). It is clear that $\operatorname{NVS~}_{h, \xi}=\mathbb{R}$. The action of the backlash operator is illustrated in Figure 3 .
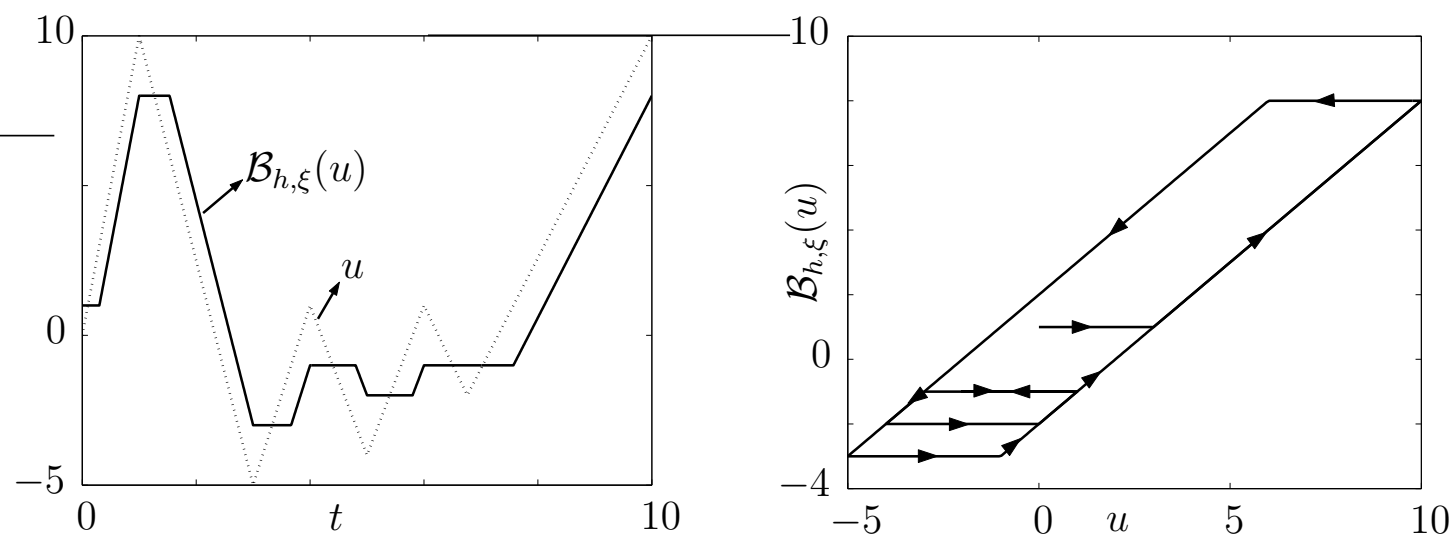

Figure 3: Backlash operator $\mathcal{B}_{h, \xi}$, with $h=2$ and $\xi=1$.

Elastic-plastic operator. The elastic-plastic operator (also called the stop operator) describes the stress-strain relation in a one-dimensional elastic-plastic element. When the modulus of the stress is smaller than the yield stress, the strain is related to the stress through Hooke's law (linear). Once the stress exceeds the yield value, it remains constant under further increasing of the strain; however, the elastic behaviour is recovered when the strain is again decreased.

For each $h \in \mathbb{R}_{+}$, define the function $e_{h}: \mathbb{R} \rightarrow \mathbb{R}$ by

$$
e_{h}(u)=\min \{h, \max \{-h, u\}\}
$$

For all $h \in \mathbb{R}_{+}$and all $\xi \in \mathbb{R}$, we introduce an operator $\mathcal{E}_{h, \xi}$ on $C_{\mathrm{pm}}\left(\mathbb{R}_{+}\right)$by defining, for every $u \in C_{\mathrm{pm}}\left(\mathbb{R}_{+}\right)$,

$$
\left(\mathcal{E}_{h, \xi}(u)\right)(t)= \begin{cases}e_{h}(u(0)-\xi) & \text { for } t=0 \\ e_{h}\left(u(t)-u\left(t_{i}\right)+\left(\mathcal{E}_{h, \xi}(u)\right)\left(t_{i}\right)\right) & \text { for } t_{i-1}<t \leq t_{i}, i \in \mathbb{N}\end{cases}
$$

where $0=t_{0}<t_{1}<t_{2}<\ldots$ is a partition of $\mathbb{R}_{+}$such that $u$ is monotone on each of the intervals $\left[t_{i}, t_{i+1}\right], i \in \mathbb{N}$. Again, $\xi$ plays the role of an "initial state". It is well known, see, for example, [3, page 44], that the operator $\mathcal{E}_{h, \xi}: C_{\mathrm{pm}}\left(\mathbb{R}_{+}\right) \rightarrow$ $C\left(\mathbb{R}_{+}\right)$can be extended uniquely to a hysteresis operator $\mathcal{E}_{h, \xi}: C\left(\mathbb{R}_{+}\right) \rightarrow C\left(\mathbb{R}_{+}\right)$; moreover, the extended operator satisfies (N1)-(N6) with $\lambda=2$ (see, for example, $\left[11\right.$, Proposition 5.7]). It is clear that NVS $\mathcal{E}_{h, \xi}=[-h, h]$. The action of the elasticplastic operator is illustrated in Figure 4. Finally, we mention that $\mathcal{E}_{h, \xi}(u)+\mathcal{B}_{h, \xi}(u)=$ $u$ for all $u \in C\left(\mathbb{R}_{+}\right)$. 

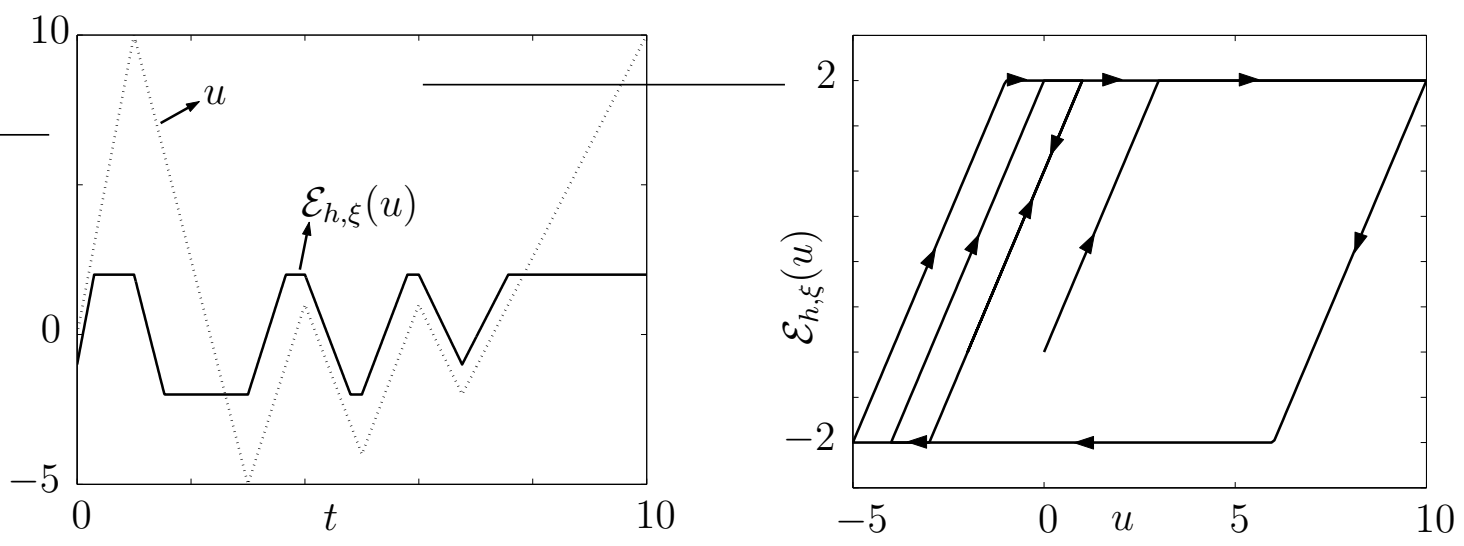

Figure 4: Elastic-plastic operator $\mathcal{E}_{h, \xi}$, with $h=2$ and $\xi=1$.

Prandtl and Preisach operators. The Preisach operator, a version of which is described below, encompasses both backlash, elastic-plastic and, more generally, Prandtl operators. It represents a far more general type of hysteresis which, for certain input functions, exhibits nested loops in the corresponding input-output characteristics. Let $\zeta: \mathbb{R}_{+} \rightarrow \mathbb{R}$ be a compactly supported and globally Lipschitz function with Lipschitz constant 1. Furthermore, let $\mu$ be a signed Borel measure on $\mathbb{R}_{+}$such that $|\mu|(K)<\infty$ for all compact sets $K \subset \mathbb{R}_{+}$, where $|\mu|$ denotes the total variation of $\mu$. Denoting the Lebesgue measure on $\mathbb{R}$ by $\mu_{L}$, let $w: \mathbb{R} \times \mathbb{R}_{+} \rightarrow \mathbb{R}$ be a locally $\left(\mu_{L} \otimes \mu\right)$-integrable function and let $w_{0} \in \mathbb{R}$. The operator $\mathcal{P}_{\zeta}: C\left(\mathbb{R}_{+}\right) \rightarrow$ $C\left(\mathbb{R}_{+}\right)$defined by

$$
\left(\mathcal{P}_{\zeta}(u)\right)(t)=\int_{0}^{\infty} \int_{0}^{\left(\mathcal{B}_{r, \zeta(r)}(u)\right)(t)} w(s, h) \mu_{L}(\mathrm{~d} s) \mu(\mathrm{d} h)+w_{0}, \quad \forall u \in C\left(\mathbb{R}_{+}\right), \quad \forall t \in \mathbb{R}_{+},
$$

is called a Preisach operator, cf. [3, p. 55]. It is clear that $\mathcal{P}_{\zeta}$ is a hysteresis operator. Under the assumption that the measure $\mu$ is finite and $w$ is essentially bounded, the operator $\mathcal{P}_{\zeta}$ is Lipschitz continuous with Lipschitz constant $|\mu|\left(\mathbb{R}_{+}\right)\|w\|_{\infty}$, see [11]. Furthermore, if we additionally assume that $\mu$ and $w$ are non-negative valued, then, as shown in [11], (N1)-(N6) hold (with $\lambda=\mu\left(\mathbb{R}_{+}\right)\|w\|_{\infty}$ in (N3)) and

$$
\begin{aligned}
& \sup \operatorname{NVS} \mathcal{P}_{\zeta}=\int_{0}^{\infty} \int_{0}^{\infty} w(s, h) \mu_{L}(\mathrm{~d} s) \mu(\mathrm{d} h)+w_{0} \in\left[w_{0}, \infty\right] \\
& \inf \operatorname{NVS} \mathcal{P}_{\zeta}=-\int_{0}^{\infty} \int_{-\infty}^{0} w(s, h) \mu_{L}(\mathrm{~d} s) \mu(\mathrm{d} h)+w_{0} \in\left[-\infty, w_{0}\right]
\end{aligned}
$$

The special case, wherein $w \equiv 1$ and $w_{0}=0$ in (8), yields the Prandtl operator

$$
\left(\mathcal{P}_{\zeta}(u)\right)(t)=\int_{0}^{\infty}\left(\mathcal{B}_{h, \zeta(h)}(u)\right)(t) \mu(\mathrm{d} h), \quad \forall u \in C\left(\mathbb{R}_{+}\right), \quad \forall t \in \mathbb{R}_{+} .
$$

It follows from (9) and (10) that the numerical value set of the Prandtl operator defined in (11) is equal to $\mathbb{R}$ (provided that $\mu \neq 0$ ). 
To provide a concrete example of a Preisach operator, we adopt a product of continuous Gaussian distributions as the weighting function $w$ (similar weighting function has been used by Mitchler et al [15] in a Preisach model of magnetic remanence in materials), that is,

$$
w:(s, h) \mapsto \frac{1}{2 \pi} \exp \left(-\left(s^{2}+h^{2}\right) / 2\right)
$$

Then

$$
\begin{array}{r}
\int_{0}^{\infty} \int_{0}^{\left(\mathcal{B}_{h, \zeta(h)}(u)\right)(t)} \exp \left(-\left(s^{2}+h^{2}\right) / 2\right) \mathrm{d} s \mathrm{~d} h=\int_{0}^{\infty} \int_{0}^{\left(\mathcal{B}_{h, \zeta(h)}(u)\right)(t)} \exp \left(-s^{2} / 2\right) \alpha(h) \mathrm{d} s \mathrm{~d} h \\
=\int_{0}^{\infty} \int_{0}^{\left(\mathcal{B}_{h, \zeta(h)}(u)\right)(t)} \exp \left(-s^{2} / 2\right) \mu_{L}(\mathrm{~d} s) \mu(\mathrm{d} h),
\end{array}
$$

where $\alpha: \mathbb{R}_{+} \rightarrow \mathbb{R}_{+}$is given by $\alpha(h)=\exp \left(-h^{2} / 2\right)$ and the measure $\mu$ is given by $\mu(\mathrm{d} h)=\alpha(h) \mathrm{d} h$. In particular, $\mu\left(\mathbb{R}_{+}\right)=\sqrt{\pi / 2}$ and the Preisach operator given by

$$
\left(\mathcal{P}_{\zeta}(u)\right)(t)=\frac{1}{2 \pi} \int_{0}^{\infty} \int_{0}^{\left(\mathcal{B}_{h, \zeta(h)}(u)\right)(t)} \exp \left(-\left(s^{2}+h^{2}\right) / 2\right) \mathrm{d} s \mathrm{~d} h+w_{0}
$$

is such that (N1)-(N6) hold, with

$$
\lambda=\frac{1}{2 \pi} \mu\left(\mathbb{R}_{+}\right) \sup _{\sigma \in \mathbb{R}_{+}} \exp (-\sigma)=\frac{1}{2 \pi} \mu\left(\mathbb{R}_{+}\right)=\frac{1}{2 \sqrt{2 \pi}}
$$

in (N3). In the case $\zeta=0$ and $w_{0}=0$, this operator is illustrated in Figure 5.
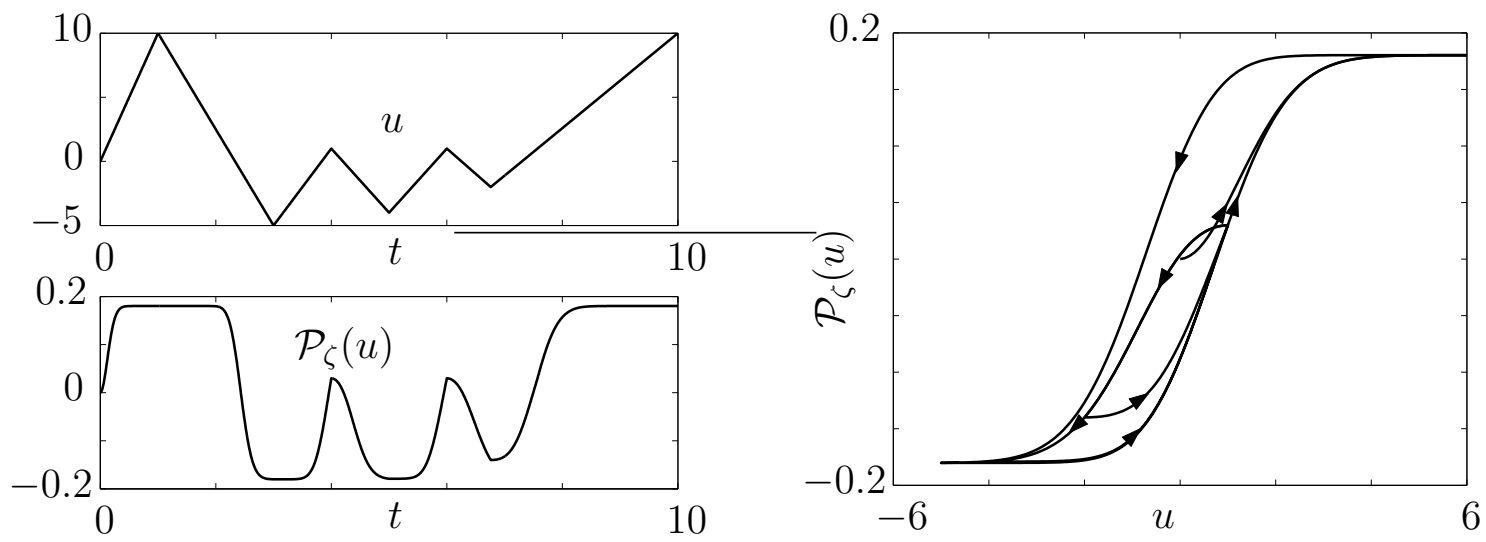

Figure 5: The Preisach operator (12) with $\zeta=0$ and $w_{0}=0$.

\section{Asymptotic behaviour of feedback systems with hysteresis}

In order to provide an appropriate analytical framework for the main investigation in Section 4 below, here we assemble some results from $[12,13]$. The first result 
pertains to a feedback interconnection of a convolution operator $G$ (with kernel $g$ ) and a hysteretic nonlinearity $\Phi$, as shown in Figure 6 below, and is contained in $[12$, Theorem 4.1].

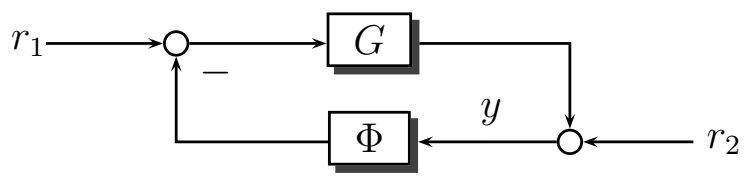

Figure 6: Feedback system with hysteretic nonlinearity

Theorem 3.1. Let $g \in L_{\alpha}^{2}\left(\mathbb{R}_{+}\right)$, for some $\alpha<0$, be a function of locally bounded variation. Let $r_{1}, r_{2} \in W_{\text {loc }}^{1,1}\left(\mathbb{R}_{+}\right)$with $r_{1}^{\prime}, r_{2}^{\prime} \in L_{\alpha}^{2}\left(\mathbb{R}_{+}\right)$. Let $\Phi: C\left(\mathbb{R}_{+}\right) \rightarrow C\left(\mathbb{R}_{+}\right)$ be a hysteresis operator satisfying (N1), (N2) and (N3) with associated constant $\lambda>0$. Assume that

$$
\inf _{\omega \in \mathbb{R}} \operatorname{Re} \mathbf{G}(i \omega)>-\frac{1}{\lambda},
$$

where $\mathbf{G}$ denotes the Laplace transform of $g$.

Then the feedback system

$$
y=g \star r_{1}+r_{2}-g \star \Phi(y),
$$

has a unique solution $y \in W_{\mathrm{loc}}^{1,1}\left(\mathbb{R}_{+}\right)$and there exist constants $\beta \in(\alpha, 0)$ and $\gamma>0$ (depending only on $g$ and $\lambda$ ) such that

$$
\begin{aligned}
\|y\|_{L^{\infty}\left(\mathbb{R}_{+}\right)}+ & \|\Phi(y)\|_{L^{\infty}\left(\mathbb{R}_{+}\right)}+\left\|y^{\prime}\right\|_{L_{\beta}^{2}\left(\mathbb{R}_{+}\right)}+\left\|(\Phi(y))^{\prime}\right\|_{L_{\beta}^{2}\left(\mathbb{R}_{+}\right)} \\
& \leq \gamma\left[\left\|r_{1}^{\prime}\right\|_{L_{\beta}^{2}\left(\mathbb{R}_{+}\right)}+\left\|r_{2}^{\prime}\right\|_{L_{\beta}^{2}\left(\mathbb{R}_{+}\right)}+\left|r_{1}(0)\right|+\left|r_{2}(0)\right|+\left|\left(\Phi\left(r_{2}\right)\right)(0)\right|\right]
\end{aligned}
$$

and $y(t)$ and $(\Phi(y))(t)$ converge to finite limits as $t \rightarrow \infty$, the convergence being exponential with rate $\beta$.

In Theorem 3.1, it is assumed that the linear component of the system is described by a convolution operator with kernel (impulse response) $g$ in $L_{\alpha}^{2}\left(\mathbb{R}_{+}\right)$for some $\alpha<0$. This implies that the linear subsystem is input-output stable and, in particular, does not contain any integrators.

The next result, Theorem 3.2, applies to a class of linear systems - containing an integrator with associated gain parameter $\kappa>0$ - of the form shown in Figure 7 , where again $G$ is a convolution operator (with kernel $g$ ), $\Phi: C\left(\mathbb{R}_{+}\right) \rightarrow C\left(\mathbb{R}_{+}\right.$) is a hysteresis operator, $r_{1}, r_{2} \in \mathbb{R}$ and $q \in L^{2}\left(\mathbb{R}_{+}\right)$.

Theorem 3.2. Let $g \in L_{\alpha}^{1}\left(\mathbb{R}_{+}\right)+\mathbb{R} \delta_{0}$ for some $\alpha<0$, where $\delta_{0}$ is unit point mass at $t=0$ (the Dirac distribution with support at $t=0$ ). Let $r_{1}, r_{2} \in \mathbb{R}, \kappa>0$ and $q \in L^{2}\left(\mathbb{R}_{+}\right)$with $\lim _{t \rightarrow \infty} q(t)=0$. Let $\Phi: C\left(\mathbb{R}_{+}\right) \rightarrow C\left(\mathbb{R}_{+}\right)$be a hysteresis operator 


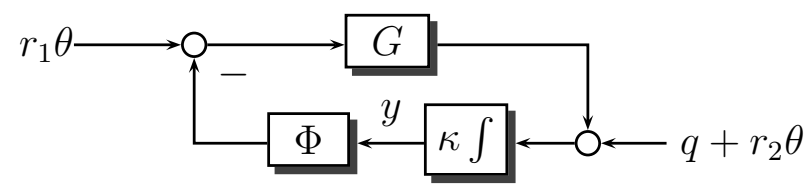

Figure 7: Feedback system with integrator and hysteretic nonlinearity

satisfying (N1)-(N6) with associated $\lambda>0$. Let $\mathbf{G}$ denote the Laplace transform of $g$ and assume that $\mathbf{G}(0)>0, r_{1}+r_{2} / \mathbf{G}(0) \in \overline{\mathrm{NVS}} \Phi$ and

$$
0<\kappa<\left\{\begin{array}{ll}
1 /(\lambda|f(\mathbf{G})|), & f(\mathbf{G}) \neq 0 \\
\infty, & f(\mathbf{G})=0,
\end{array} \quad \text { where } f(\mathbf{G}):=\inf _{\omega \in \mathbb{R}^{*}} \operatorname{Re}\left(\frac{\mathbf{G}(i \omega)}{i \omega}\right) .\right.
$$

Then, for each $y_{0} \in \mathbb{R}$, the initial-value problem (describing the feedback system shown in Figure 7)

$$
\dot{y}=\kappa\left(r_{1} g \star \theta+q+r_{2} \theta-g \star \Phi(y)\right), \quad y(0)=y_{0} \in \mathbb{R},
$$

has a unique solution $y \in W_{\mathrm{loc}}^{1,1}\left(\mathbb{R}_{+}\right)$and $y$ has the following properties: $\lim _{t \rightarrow \infty} \dot{y}(t)=$ $0, \lim _{t \rightarrow \infty}(\Phi(y))(t)=r_{1}+r_{2} / \mathbf{G}(0)$ and $(\Phi(y))^{\prime} \in L^{2}\left(\mathbb{R}_{+}\right)$. Furthermore, if $r_{1}+$ $r_{2} / \mathbf{G}(0)$ is an interior point of $\operatorname{NVS} \Phi$, then $y$ is bounded.

Proof. Since $g \in L_{\alpha}^{1}\left(\mathbb{R}_{+}\right)+\mathbb{R} \delta_{0}$, with $\alpha<0$, it follows that

$$
\limsup _{s \rightarrow 0, \operatorname{Re} s>0}\left|\frac{\mathbf{G}(s)-\mathbf{G}(0)}{s}\right|=\left|\mathbf{G}^{\prime}(0)\right|<\infty .
$$

Hence, $\mathbf{G}$ satisfies the condition $(\mathbf{L})$ in $[13$, Theorem 4.1]. Note that $g \star \theta-\mathbf{G}(0) \theta \in$ $L^{2}\left(\mathbb{R}_{+}\right)$and $(g \star \theta)(t)-\mathbf{G}(0) \rightarrow 0$ as $t \rightarrow \infty$. Furthermore, observe that system (17) has the same form as the system in [13, (4.1)], with $h=0, \vartheta=0$ and $\psi=$ id in the latter and with the roles of $\rho$ and $g$ in the latter being played, respectively, by $\mathbf{G}(0) r_{1}+r_{2}$ and $r_{1}(\mathbf{G}(0)-g \star \theta)-q \in L^{2}\left(\mathbb{R}_{+}\right)$in the present context. Therefore, Theorem 4.1 in [13] may be applied to establish that (17) has a unique solution $y \in W_{\mathrm{loc}}^{1,1}\left(\mathbb{R}_{+}\right)$, and $\lim _{t \rightarrow \infty} \dot{y}(t)=0,(\Phi(y))^{\prime} \in L^{2}\left(\mathbb{R}_{+}\right)$and $\lim _{t \rightarrow \infty}(\Phi(y))(t)=: \Phi^{\infty}$ exists. Moreover, Theorem 4.1 in [13] also shows that $y$ is bounded, provided that $r_{1}+r_{2} / \mathbf{G}(0)$ is an interior point of $\operatorname{NVS} \Phi$. It remains to show that $\Phi^{\infty}=r_{1}+$ $r_{2} / \mathbf{G}(0)$. This follows easily from (17) and the facts that $\dot{y}(t) \rightarrow 0,(g \star \theta)(t) \rightarrow \mathbf{G}(0)$ and $(g \star \Phi(y))(t) \rightarrow \mathbf{G}(0) \Phi^{\infty}$ as $t \rightarrow \infty$.

\section{$4 \quad$ PID control of systems with hysteresis}

We now focus attention on the application of Theorems 3.1 and 3.2 in the analysis and design of PID control in the context of each of system structures (1) and (2). 


\subsection{Systems of form (1)}

Consider again a second-order system described by (1):

$$
m \ddot{x}+c \dot{x}+\Phi(x)=u+d \theta, \quad x(0)=x_{0}, \quad \dot{x}(0)=v_{0}, \quad m>0, c>0, d \in \mathbb{R},
$$

where $d \theta$ is a constant disturbance signal. Assume that $r \in \mathbb{R}$ is a constant reference signal, in which case, the control objective is to determine, by feedback, the control input $u$ to achieve the tracking objective: $x(t) \rightarrow r$ as $t \rightarrow \infty$. We will investigate the efficacy of the following PID control in achieving this objective:

$$
u(t)=-k_{p}(x(t)-r)-k_{d} \dot{x}(t)-k_{i} \int_{0}^{t}(x(\tau)-r) \mathrm{d} \tau+u_{0},
$$

where $u_{0} \in \mathbb{R}$ is the initial condition of the integrator and $k_{p}, k_{d}, k_{i} \geq 0$ are suitably chosen gains. Let $0<\omega \leq \infty$. A function $x \in C^{2}([0, \omega))$ satisfying (18) and (19) is said to be a solution on $[0, \omega)$ of the closed-loop system given by (18) and (19).

Theorem 4.1. Let $\Phi: C\left(\mathbb{R}_{+}\right) \rightarrow C\left(\mathbb{R}_{+}\right)$be a hysteresis operator satisfying (N1), (N2) and (N3) (with associated constant $\lambda>0$ ). Let $r, d \in \mathbb{R}$. If $k_{p}, k_{d}, k_{i}$ are chosen such that

(A1) $k_{d}>-c+\sqrt{\left(2 k_{p}+\lambda\right) m}$

(A2) $0<k_{i}<k_{p}\left(k_{p}+\lambda\right) /\left(2\left(c+k_{d}\right)\right)$,

then, for each $\left(x_{0}, v_{0}, u_{0}\right) \in \mathbb{R}^{3}$, the initial-value problem given by (18) and (19) has a unique solution $x \in C^{2}\left(\mathbb{R}_{+}\right), x(t) \rightarrow r, \dot{x}(t) \rightarrow 0$ and $\ddot{x}(t) \rightarrow 0$ as $t \rightarrow \infty$; moreover, $(\Phi(x))(t)$ converges to a finite limit as $t \rightarrow \infty$ (all convergences being exponentially fast).

Proof. By standard arguments (as in, for example, [12]), there exists a unique solution $x \in C^{2}\left(\mathbb{R}_{+}\right)$of the initial-value problem given by (18) and (19). Setting

$$
z(t):=\int_{0}^{t}(x(\tau)-r) \mathrm{d} \tau-\frac{1}{k_{i}}\left(u_{0}+d\right), \quad \forall t \in \mathbb{R}_{+}
$$

and $p(s):=m s^{3}+\left(c+k_{d}\right) s^{2}+k_{p} s+k_{i}$, it follows that

$$
p\left(\frac{\mathrm{d}}{\mathrm{d} t}\right) z=-\Phi(\dot{z}+r \theta) .
$$

Let $h \in C^{3}\left(\mathbb{R}_{+}\right)$and $\rho \in C^{3}\left(\mathbb{R}_{+}\right)$be the unique solutions of the following two initial-value problems

$$
p\left(\frac{\mathrm{d}}{\mathrm{d} t}\right) h=0, \quad h(0)=0, \dot{h}(0)=0, \ddot{h}(0)=\frac{1}{m}
$$


and

$p\left(\frac{\mathrm{d}}{\mathrm{d} t}\right) \rho=0, \rho(0)=z(0)=-\frac{u_{0}+d}{k_{i}}, \dot{\rho}(0)=\dot{z}(0)=x(0)-r, \ddot{\rho}(0)=\ddot{z}(0)=\dot{x}(0)$,

respectively. Using the variation-of-parameters formula for higher order systems, we can rewrite (20) in the form

$$
z=-h \star \Phi(\dot{z}+r \theta)+\rho .
$$

Clearly $x=\dot{z}+r \theta$. Differentiating (21), writing $g:=\dot{h}$ and using the fact that $h(0)=0$, yields

$$
x=\dot{\rho}+r \theta-g \star \Phi(x),
$$

which has the structure of (14) (with $r_{1}=0$ and $r_{2}=\dot{\rho}+r \theta$ ) to which Theorem 3.1 potentially applies. First, however, we need to show that the hypotheses of Theorem 3.1 hold in the context of $(22)$. Clearly, $\dot{\rho}+r \theta \in W_{\text {loc }}^{1,1}\left(\mathbb{R}_{+}\right)$, so it remains to show that

$$
\text { (a) } g, \ddot{\rho} \in L_{\alpha}^{2} \text { for some } \alpha<0, \quad \text { (b) } \inf _{\omega \in \mathbb{R}} \operatorname{Re} \mathbf{G}(i \omega)>-1 / \lambda,
$$

where $\mathbf{G}$ is the Laplace transform of $g$, that is,

$$
\mathbf{G}(s)=\frac{s}{m s^{3}+\left(c+k_{d}\right) s^{2}+k_{p} s+k_{i}}=\frac{s}{p(s)} .
$$

To conclude (a), it suffices to show that $p$ is Hurwitz, in which case $h, \dot{h}=g, \ddot{h}=\dot{g}$, $\rho, \dot{\rho}$ and $\ddot{\rho}$ are exponential decaying functions. By the Routh-Hurwitz criterion, $p$ is Hurwitz if, and only if,

$$
c+k_{d}>0 \quad \text { and } \quad 0<k_{i}<k_{p}\left(c+k_{d}\right) / m .
$$

Clearly (A1) implies the first of inequalities (23) and, moreover, implies that $\left(2 k_{p}+\right.$ $\lambda) /\left(c+k_{d}\right)<\left(c+k_{d}\right) / m$ which, together with (A2), gives the second of inequalities $(23)$ :

$$
0<k_{i}<\frac{k_{p}\left(k_{p}+\lambda\right)}{2\left(c+k_{d}\right)}<\frac{k_{p}\left(2 k_{p}+\lambda\right)}{c+k_{d}}<\frac{k_{p}\left(c+k_{d}\right)}{m} .
$$

It remains to show that (b) holds. First observe that

$$
\begin{aligned}
\inf _{\omega \in \mathbb{R}} \operatorname{Re} & \left(\frac{i \omega}{m(i \omega)^{3}+\left(c+k_{d}\right)(i \omega)^{2}+k_{p}(i \omega)+k_{i}}\right)>-\frac{1}{\lambda} \\
& \Leftrightarrow \inf _{\omega \in \mathbb{R}} \frac{k_{p} \omega^{2}-m \omega^{4}}{\left(k_{p} \omega-m \omega^{3}\right)^{2}+\left(k_{i}-\left(c+k_{d}\right) \omega^{2}\right)^{2}}>-\frac{1}{\lambda} \\
& \Leftrightarrow\left(k_{p} \omega-m \omega^{3}\right)^{2}+\lambda\left(k_{p} \omega^{2}-m \omega^{4}\right)+\left(k_{i}-\left(c+k_{d}\right) \omega^{2}\right)^{2}>0 \quad \forall \omega \in \mathbb{R} \\
& \Leftrightarrow m^{2} \omega^{6}+\beta \omega^{4}+\left(k_{p}^{2}+\lambda k_{p}-2 k_{i}\left(c+k_{d}\right)\right) \omega^{2}+k_{i}^{2}>0 \quad \forall \omega \in \mathbb{R} \\
& \Leftrightarrow m^{2} \xi^{3}+\beta \xi^{2}+\left(k_{p}^{2}+\lambda k_{p}-2 k_{i}\left(c+k_{d}\right)\right) \xi+k_{i}^{2}>0 \quad \forall \xi \in \mathbb{R}_{+},
\end{aligned}
$$

where $\beta:=\left(c+k_{d}\right)^{2}-\left(2 k_{p}+\lambda\right) m$. Now observe that the inequality (24) holds since, by (A1) and (A2), the polynomial in $\xi$ on the LHS of (24) has positive coefficients, 
whence hypothesis (b) is satisfied. All hypotheses of Theorem 3.1 are now in place (in the context of system (22)), and so we may conclude that $x(t)$ and $(\Phi(x))(t)$ converge to finite limits as $t \rightarrow \infty$, the convergence being exponentially fast. Since $h, \rho$ are exponentially decaying functions and since $(\Phi(x))(t)=(\Phi(\dot{z}+r \theta))(t)$ converges exponentially, as $t \rightarrow \infty$, to a finite limit, we conclude from (21) that $z(t)$ also converges, as $t \rightarrow \infty$, to a finite limit at exponential rate. Consequently, since both $z(t)=\int_{0}^{t}(x(\tau)-r) \mathrm{d} \tau-\left(u_{0}+d\right) / k_{i}$ and $x(t)-r$ have finite limits as $t \rightarrow$ $\infty$, and converge exponentially, we conclude that $x(t)-r \rightarrow 0$ exponentially as $t \rightarrow \infty$. A routine argument involving the differentiation of (22) now shows that $\dot{x}(t) \rightarrow 0$ exponentially as $t \rightarrow \infty$. Consequently, the control signal $u(t)$ given by (19) converges exponentially fast to a finite limit as $t \rightarrow \infty$. Finally, invoking (18), we obtain that, $\ddot{x}(t)$ converges exponentially fast to a finite limit as $t \rightarrow \infty$. Boundedness of $\dot{x}$ shows that this limit is equal to 0 .

Remark 4.2. Assume that the parameters $m, c$ and $\lambda$ are unknown, but belong to known intervals, viz. $m \in\left(0, m_{+}\right], c \in\left[c_{-}, c_{+}\right]$and $\lambda \in\left(0, \lambda_{+}\right]$, where $m_{+}>0$, $0<c_{-} \leq c_{+}$and $\lambda_{+}>0$ are known constants. If the PID controller gains are determined by using the following procedure:

(P1) let $k_{p}>0$ be arbitrary,

(P2) choose $k_{d}$ such that $k_{d}>-c_{-}+\sqrt{\left(2 k_{p}+\lambda_{+}\right) m_{+}}$,

(P3) choose $k_{i}$ such that $0<k_{i}<k_{p}^{2} /\left(2\left(c_{+}+k_{d}\right)\right)$,

then (A1) and (A2) hold and Theorem 4.1 applies to conclude that the PID controller, with the above choice of gain, solves the tracking and disturbance rejection problem

\subsection{Systems of form (2)}

In this subsection we consider second-order systems described by (2):

$$
\left.\begin{array}{l}
m \ddot{x}+c \dot{x}+k x=d_{1} \theta+\Phi\left(u+d_{2} \theta\right), \quad x(0)=x_{0}, \quad \dot{x}(0)=v_{0}, \\
m>0, c>0, k>0, d_{1}, d_{2} \in \mathbb{R} .
\end{array}\right\}
$$

where $d_{1} \theta$ and $d_{2} \theta$ are constant disturbance signals. We will investigate the efficacy of the control structure (19) in both the absence $\left(k_{d}=0\right)$ and presence $\left(k_{d}>0\right)$ of derivative feedback.

Theorem 4.3. Let $\Phi: C\left(\mathbb{R}_{+}\right) \rightarrow C\left(\mathbb{R}_{+}\right)$be a hysteresis operator satisfying (N1)(N6) with associated $\lambda>0$. Let $r, d_{1}, d_{2} \in \mathbb{R}$ and assume that $r k-d_{1} \in \overline{\operatorname{NVS}} \Phi$.

Case (a). Set $k_{d}=0$ and let $k_{p}, k_{i}>0$ be chosen such that:

(A) $0<k_{i}<\frac{k_{p} k}{c}<\frac{c k}{\lambda m}$. 
Case (b). Let $k_{p}, k_{i}, k_{d}>0$ be chosen such that:

(B1) $0<k_{i}<\infty$

(B2) $k_{p}>\frac{c k_{i}}{k}$

(B3) $k_{d}>\frac{m k_{p}}{c}$.

Then there exists a unique solution $x \in C^{2}\left(\mathbb{R}_{+}\right)$of the closed-loop system given by (19) and (25), and

$$
\lim _{t \rightarrow \infty} x(t)=r, \quad \lim _{t \rightarrow \infty} \dot{x}(t)=0, \quad \lim _{t \rightarrow \infty} \ddot{x}(t)=0, \quad \lim _{t \rightarrow \infty}\left(\Phi\left(u+d_{2} \theta\right)\right)(t)=r k-d_{1} .
$$

Moreover, if $r k-d_{1}$ is an interior point of $\operatorname{NVS} \Phi$, then the control signal u given by (19) is bounded.

Proof. By standard arguments (as in, for example, [12]), there exists a unique solution $x \in C^{2}\left(\mathbb{R}_{+}\right)$of the closed-loop system given by (19) and (25). Set $p(s):=$ $m s^{2}+c s+k$, let $h$ be the solution of the initial-value problem

$$
p\left(\frac{\mathrm{d}}{\mathrm{d} t}\right) h=0, \quad h(0)=0, \quad \dot{h}(0)=\frac{1}{m},
$$

and let $\rho$ be the solution of the initial-value problem

$$
p\left(\frac{\mathrm{d}}{\mathrm{d} t}\right) \rho=0, \quad \rho(0)=x_{0}, \quad \dot{\rho}(0)=v_{0} .
$$

Then the solution $x$ of the closed-loop system given by (19) and (25) satisfies

$$
x=h *\left[\Phi\left(-k_{p}(x-r \theta)-k_{d} \dot{x}-k_{i} \int_{0}^{\cdot}(x(\tau)-r) \mathrm{d} \tau+\left(u_{0}+d_{2}\right) \theta\right)+d_{1} \theta\right]+\rho .
$$

Define $w \in C^{1}\left(\mathbb{R}_{+}\right)$by

$$
\begin{aligned}
w(t) & :=u(t)+d_{2} \\
& =-k_{p}(x(t)-r)-k_{d} \dot{x}(t)-k_{i} \int_{0}^{t}(x(\tau)-r) \mathrm{d} \tau+u_{0}+d_{2}, \quad \forall t \geq 0,
\end{aligned}
$$

which, together with $(26)$ and the facts that $h(0)=0$ and $\dot{h}(0)=1 / m$, implies

$$
\begin{aligned}
\dot{w} & =-k_{p} \dot{x}-k_{d} \ddot{x}-k_{i}(x-r \theta) \\
& =k_{i}\left(r \theta+q-d_{1} g \star \theta-g \star \Phi(w)\right), \quad w(0)=-k_{p}\left(x_{0}-r\right)-k_{d} v_{0}+u_{0}+d_{2},
\end{aligned}
$$

where

$$
g:=\frac{k_{d}}{k_{i}}\left[\ddot{h}+\frac{1}{m} \delta_{0}\right]+\frac{k_{p}}{k_{i}} \dot{h}+h
$$


$\delta_{0}$ being the unit point mass at 0 (the Dirac distribution with support at $t=0$ ), and

$$
q:=-\frac{k_{d}}{k_{i}} \ddot{\rho}-\frac{k_{p}}{k_{i}} \dot{\rho}-\rho .
$$

Noting that (28) has the structure of (17) (with the roles of $y, \kappa, r_{1}$ and $r_{2}$ being played by $w, k_{i},-d_{1}$ and $r$, respectively), we seek to apply Theorem 3.2 by showing that all hypotheses therein hold in the present context. Since $m, c, k, k_{i}, k_{p}>0$ and $k_{d} \geq 0$, it follows that $h, \dot{h}, \ddot{h}$ and $q$ are exponentially decaying functions. Therefore, $g \in L_{\alpha}^{1}\left(\mathbb{R}_{+}\right)+\mathbb{R} \delta_{0}$, for some $\alpha<0$. Moreover, the Laplace transform of $g$ is given by

$$
\mathbf{G}(s)=\frac{1}{k_{i}} \frac{k_{d} s^{2}+k_{p} s+k_{i}}{m s^{2}+c s+k} .
$$

Since $k>0$, we have $\mathbf{G}(0)=1 / k>0$. By assumption, $r k-d_{1} \in \overline{\mathrm{NVS}} \Phi$, and we see that the hypothesis of Theorem 3.2 involving $\overline{N V S} \Phi$ is satisfied.

It remains to show show that hypothesis (16) of Theorem 3.2 holds (with $\kappa$ replaced by $k_{i}$ ). To this end, observe that

$$
\begin{aligned}
f(\mathbf{G})=\inf _{\omega \in \mathbb{R}^{*}} \operatorname{Re}\left(\frac{\mathbf{G}(i \omega)}{i \omega}\right) & =\inf _{\omega \in \mathbb{R}^{*}} \operatorname{Re}\left(\frac{k_{d}(i \omega)^{2}+k_{p} i \omega+k_{i}}{k_{i}\left(m(i \omega)^{3}+c(i \omega)^{2}+k i \omega\right)}\right) \\
& =\inf _{\omega \in \mathbb{R}}\left(\frac{\left(k k_{p}-c k_{i}\right)+\omega^{2}\left(c k_{d}-m k_{p}\right)}{k_{i}\left(m^{2} \omega^{4}+\left(c^{2}-2 m k\right) \omega^{2}+k^{2}\right)}\right) \\
& =\inf _{\xi \in \mathbb{R}_{+}}\left(\frac{\left(k k_{p}-c k_{i}\right)+\xi\left(c k_{d}-m k_{p}\right)}{k_{i}\left((m \xi-k)^{2}+c^{2} \xi\right)}\right) .
\end{aligned}
$$

Define $\zeta: \mathbb{R}_{+} \rightarrow \mathbb{R}$ by

$$
\zeta(\xi)=\frac{\left(k k_{p}-c k_{i}\right)+\xi\left(c k_{d}-m k_{p}\right)}{k_{i}\left((m \xi-k)^{2}+c^{2} \xi\right)} .
$$

We consider Case (a) and Case (b) separately.

Case (a). Let $k_{d}=0$ and let (A) hold. Then we have $k k_{p}-c k_{i}>0$ and so

$$
0>f(\mathbf{G})=\inf _{\xi \in \mathbb{R}_{+}} \zeta(\xi) \geq \inf _{\xi \in \mathbb{R}_{+}} \hat{\zeta}(\xi), \quad \text { where } \hat{\zeta}(\xi):=\frac{-m k_{p} \xi}{k_{i}\left((m \xi-k)^{2}+c^{2} \xi\right)} .
$$

It is readily verified that $\hat{\zeta}$ attains a global minimum over $\mathbb{R}_{+}$at $\xi=k / m$. Therefore,

$$
0>f(\mathbf{G}) \geq \hat{\zeta}(k / m)=-\frac{m k_{p}}{c^{2} k_{i}} .
$$

Moreover, by (A), $1<c^{2} /\left(\lambda m k_{p}\right)$ and so

$$
k_{i}<\frac{k_{i} c^{2}}{\lambda m k_{p}} \leq \frac{1}{\lambda|f(\mathbf{G})|} .
$$


Case (b). Let $k_{p}, k_{i}, k_{d}>0$ be such that (B1), (B2) and (B3) hold. In this case, we have $k k_{p}-c k_{i}>0$ and $c k_{d}-m k_{p}>0$. Therefore, $\zeta(\xi) \geq 0$ for all $\xi \in \mathbb{R}_{+}$and

$$
f(\mathbf{G})=\lim _{\xi \rightarrow \infty} \zeta(\xi)=0 .
$$

Therefore, in each of cases (a) and (b), hypothesis (16) of Theorem 3.2 holds. As a consequence, we may apply Theorem 3.2 to conclude that there exists a unique solution $w \in W_{\text {loc }}^{1,1}\left(\mathbb{R}_{+}\right)$of (28) with the following properties: $\dot{w}(t) \rightarrow 0$ and $(\Phi(w))(t) \rightarrow r k-d_{1}$ as $t \rightarrow \infty$. Next, we show that $e(t):=x(t)-r \rightarrow 0$ and $\dot{e}(t)=\dot{x}(t) \rightarrow 0$ as $t \rightarrow \infty$. Indeed, by $(27)$,

$$
k_{d} \ddot{e}+k_{p} \dot{e}+k_{i} e=-\dot{w} .
$$

Since $k_{p}, k_{i}>0$ and $k_{d} \geq 0$, the system described by (31) (with $-\dot{w}$ as input) is asymptotically stable. Consequently, since $\dot{w}(t) \rightarrow 0$ as $t \rightarrow \infty$, we may conclude that $e(t) \rightarrow 0$ and $\dot{e}(t)=\dot{x}(t) \rightarrow 0$ as $t \rightarrow \infty$. It now follows from (25) that $\ddot{x}(t) \rightarrow 0$ as $t \rightarrow \infty$. Finally, if $r k-d_{1}$ is in the interior of $\operatorname{NVS} \Phi$, then boundedness of $w$, and hence of $u$, follows from the last part of Theorem 3.2.

Remark 4.4. (i) Assume that the parameters $m, c, k$ and $\lambda$ are unknown, but belong to known intervals:

$$
m \in\left(0, m_{+}\right], \quad c \in\left[c_{-}, c_{+}\right], \quad k \in\left[k_{-}, \infty\right), \quad \text { and } \quad \lambda \in\left(0, \lambda_{+}\right]
$$

where $m_{+}, c_{-}, c_{+}, k_{-}$and $\lambda_{+}$are known positive constants. We give procedures for choosing the PID-controller gains in terms of the constants $m_{+}, c_{-}, c_{+}, k_{-}$and $\lambda_{+}$.

Case (a). Set $k_{d}=0$. If the gains $k_{p}, k_{i}$ of the PI controller are determined by the following procedure:

(PA) choose $k_{p}, k_{i}$ such that

$$
0<k_{i}<\frac{k_{p} k_{-}}{c_{+}}<\frac{c_{-}^{2} k_{-}}{\lambda_{+} m_{+} c_{+}},
$$

then (A) holds and Theorem 4.3 applies to conclude that the PI controller, with the above gain selection, solves the tracking and disturbance rejection problem.

Case (b). In the case of PID control, if the controller gains are determined by the following procedure:

(PB1) choose $0<k_{i}<\infty$,

(PB2) choose $k_{p}>0$ such that $k_{p}>c_{+} k_{i} / k_{-}$,

(PB3) choose $k_{d}>0$ such that $k_{d}>m_{+} k_{p} / c_{-}$,

then (B1), (B2) and (B3) hold and Theorem 4.3 applies to conclude that the PID controller, with the above gain selection, solves the tracking and disturbance rejection problem. 
(ii) In general $d_{1}$ is unknown (as is $d_{2}$ ), but it is reasonable to assume that $d_{1} \in$ $\left[d_{1}^{-}, d_{1}^{+}\right]$, where $d_{1}^{-}$and $d_{1}^{+}$are known. Moreover, it is reasonable to assume that $k \in\left[k_{-}, k_{+}\right]$, where $0<k_{-}<k_{+}$are known constants. The conditions

$$
\begin{array}{ll}
r k_{-}-d_{1}^{+}, r k_{+}-d_{1}^{-} \in \overline{\operatorname{NVS}} \Phi, & \text { if } r \geq 0, \\
r k_{+}-d_{1}^{+}, r k_{-}-d_{1}^{-} \in \overline{\operatorname{NVS}} \Phi, & \text { if } r<0
\end{array}
$$

are sufficent conditions in terms of $d_{1}^{-}, d_{1}^{+}, k_{-}$and $k_{+}$guaranteeing that $r k-d_{1} \in$ $\overline{\mathrm{NVS}} \Phi$ for all $d_{1} \in\left[d_{1}^{-}, d_{1}^{+}\right]$and all $k \in\left[k_{-}, k_{+}\right]$.

\section{$5 \quad$ Examples}

In this section, we illustrate our main results in the context of Prandtl operators, as discussed in Section 2. In particular, recalling (11), we consider the hysteresis operator $\mathcal{P}_{0}: C\left(\mathbb{R}_{+}\right) \rightarrow C\left(\mathbb{R}_{+}\right)$defined by

$$
\begin{aligned}
\left(\mathcal{P}_{0}(u)\right)(t) & =\int_{0}^{\infty}\left(\mathcal{B}_{h, 0}(u)\right)(t) \chi_{[0, l]} \mathrm{d} h \\
& =\int_{0}^{l}\left(\mathcal{B}_{h, 0}(u)\right)(t) \mathrm{d} h, \quad \forall u \in C\left(\mathbb{R}_{+}\right), \quad \forall t \in \mathbb{R}_{+},
\end{aligned}
$$

where $l>0$ is a positive constant and $\chi_{[0, l]}$ is the indicator function of the interval $[0, l]$. This operator satisfies (N1)-(N6) (with $\lambda=l$ in (N3)), has numerical value set $\operatorname{NVS}_{\mathcal{P}_{0}}=\mathbb{R}$, and exhibits nested loops as depicted in Figure 8.
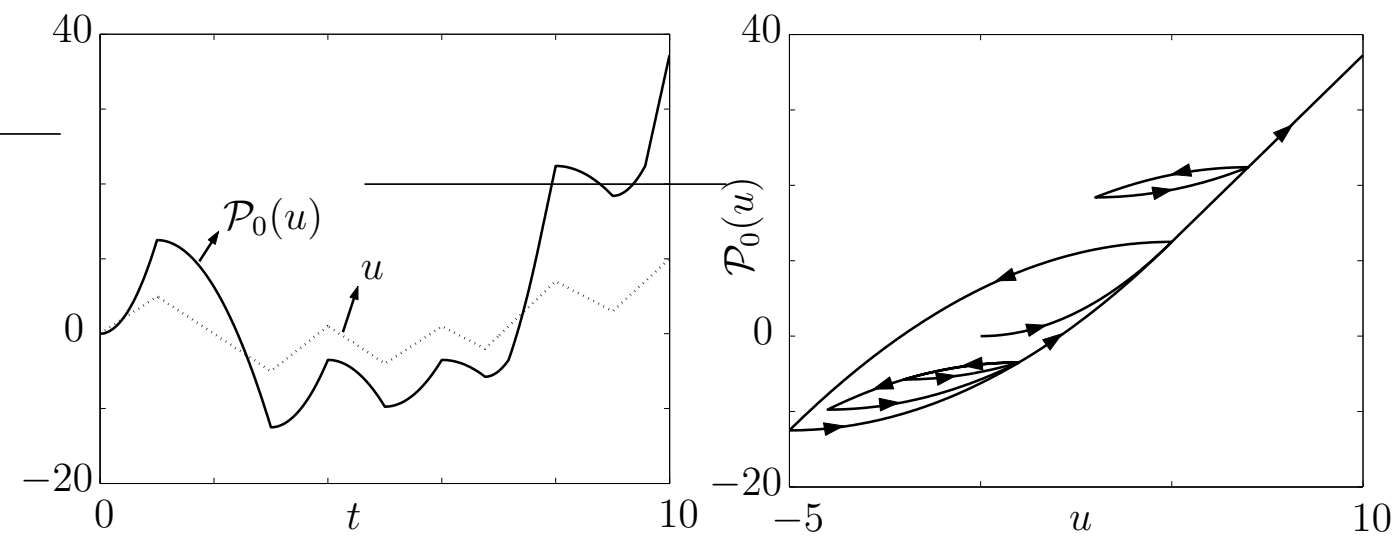

Figure 8: Behaviour of the hysteresis operator $\mathcal{P}_{0}$ with $l=5$.

\subsection{Systems of form (1)}

Consider system (18) with $\Phi=\mathcal{P}_{0}$ and with $m \in(0,2], c \in[1,3]$ and $l=\lambda \in$ $(0,10]$. Assume a constant disturbance $d=1$, reference signal $r=1$ and zero initial 
condition $u_{0}=0$ on the integrator. Using the procedure in Remark 4.2, the gains of PID controller are chosen as follows: $k_{p}=10, k_{d}=8, k_{i}=4$. For nominal plant parameters values $m=1, c=2$ and $\lambda=5$, Figure 9 shows the evolution of the closed-loop system with zero initial state.

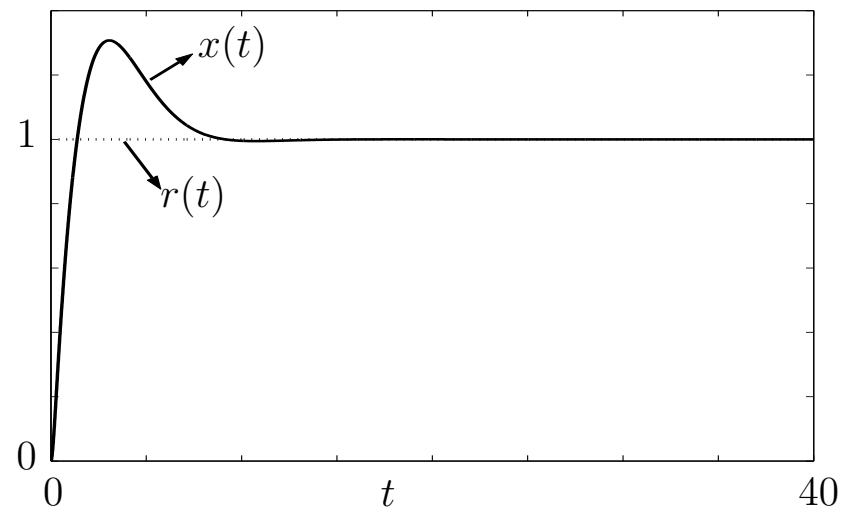

Figure 9: System (1) under PID control.

With appropriate modifications (viz re-initialization of the problem at points of discontinuity of the reference signal), our analysis extends to the problem of tracking piecewise constant signals. Consider, for example, a periodic function $r$ with period 40 and $r(t) \in\{0,1\}$ for all $t$. With nominal plant parameter values and controller gain values as above, Figure 10 illustrates system behaviour under PID control.

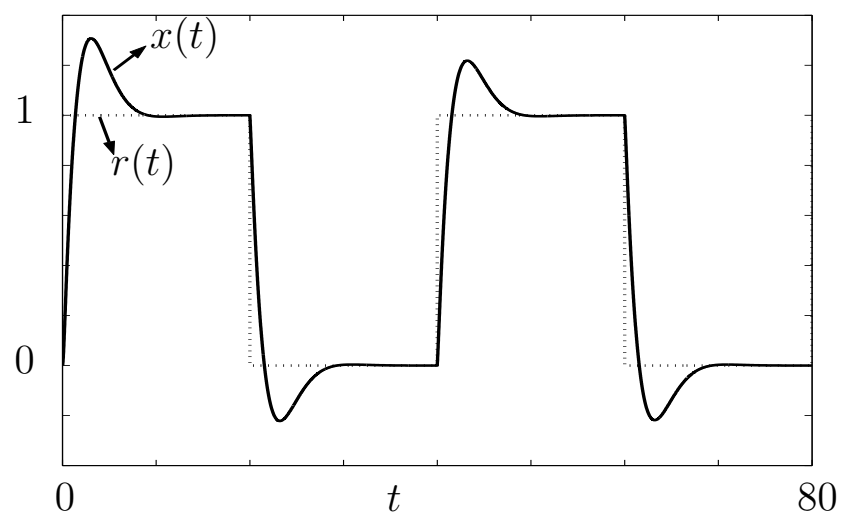

Figure 10: System (1), with periodic reference signal $r$, under PID control.

\subsection{Systems of form (2)}

Finally, consider system (25) with $\Phi$ as above and with $m \in(0,2], c \in[1,3]$, $\lambda \in(0,10]$ and $k \geq 4$. Assume a constant disturbance $d_{1}=1$, reference signal $r=1$, zero disturbance $d_{2}=0$ and $u_{0}=0$. 
Case (a). Set $k_{d}=0$. Using the procedure in Case (a) of Remark 4.4, the gains of PI controller are chosen as follows: $k_{p}=0.04, k_{i}=0.05$. For nominal plant parameters values $m=1, c=2, k=4$ and $\lambda=5$, Figure 11 shows the evolution of the closed-loop system with zero initial state: as $t \rightarrow \infty, x(t)$ converges (albeit slowly) to the constant reference signal $r$ as predicted by Theorem 4.3.

Case (b). We now include derivative feedback action $\left(k_{d}>0\right)$. In this case, a PID controller is used instead of PI controller. Using the procedure in Case (b) of Remark 4.4, the gains of PID controller may be chosen as follows: $k_{i}=10, k_{p}=10$ and $k_{d}=30$. Again, with nominal plant parameter values $m=1, c=2, k=4$ and $\lambda=5$, Figure 11 shows the evolution of the closed-loop system with zero initial state. It can be seen from this figure that, although the displacement $x(t)$ converges asymptotically to the constant reference signal $r$ under either PI or PID control, the PID controller generates the faster response: this is not unexpected given the presence of derivative feedback action in the latter. Finally, we illustrate the case

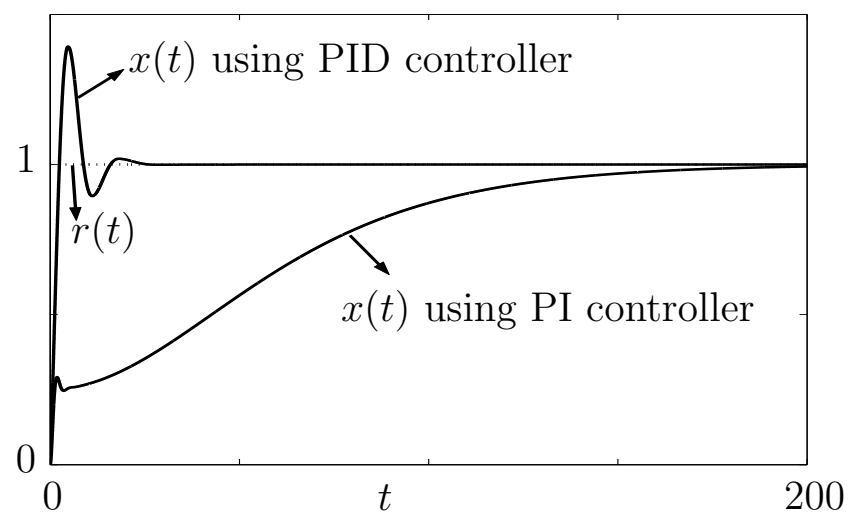

Figure 11: System (2) under PI and PID control.

of a periodic reference signal $r$ with period 100 and $r(t) \in\{0,1\}$ for all $t$. For the nominal plant parameter values and PID controller gains as above, Figure 12 depicts the system behaviour under PID control.

\section{References}

[1] J.M.T.A. Adriaens, W.L. de Koning, R. Banning, "Design and modeling of a piezoactuated positioning mechanism," Proc. 36th IEEE Conf. Decision Contr., pp. 19781983, San Diego, 1997.

[2] F. Al-Bender, W. Symens, J. Swevers, H. Van Brussle, "Theoretical analysis of the dynamic behaviour of hysteresis elements in mechanical systems", Int. J. Non-Linear Mechanics, vol. 39, pp. 1721-1735, 2004.

[3] M. Brokate, J. Sprekels, Hysteresis and Phase Transitions, Springer Verlag, New York, 1996. 


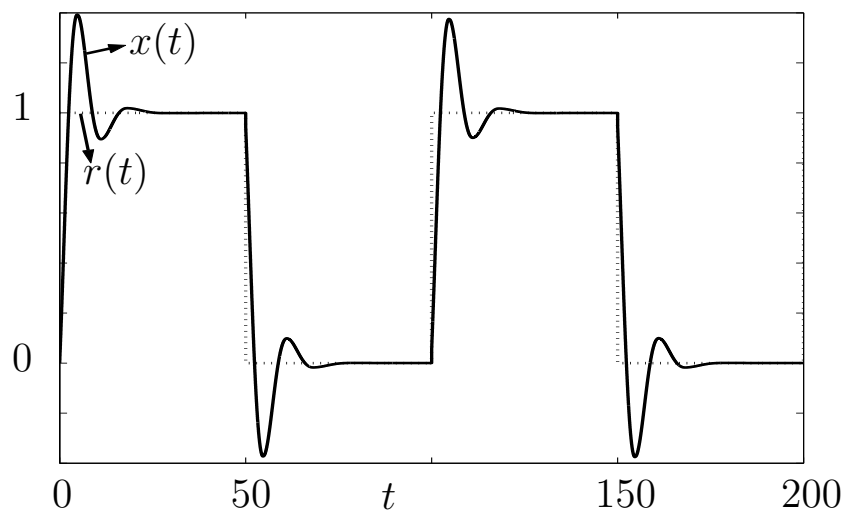

Figure 12: System (2), with periodic reference signal $r$, under PID control.

[4] P. Ge, M. Jouaneh, "Tracking control of a piezoceramic actuator, " IEEE Trans. Contr. Syst. Techn., vol. 4, pp. 209-216, 1996.

[5] R.B. Gorbet, K.A. Morris, "Closed-loop position control of Preisach hysteresis", Journal of Intelligent Material Systems and Structures, vol. 14, pp. 483-495, 2003.

[6] R.B. Gorbet, K.A. Morris, D.W.L. Wang, "Passivity-based stability and control of hysteresis in smart actuators," IEEE Trans. Contr. Syst. Techn., vol. 9, pp. 5-16, 2001.

[7] F. Ikhouane, J. Rodellar, "A linear controller for hysteretic systems," IEEE Trans. Automat. Contr., vol. 51, pp. 340-344, 2006.

[8] F. Ikhouane, V. Mañosa, J. Rodellar, "Dynamic properties of the hysteretic BoucWen model," Systems \& Control Letters, vol. 56, pp. 197-205, 2007.

[9] R.V. Iyer, X. Tan, P.S. Krishnaprasad, "Approximate inversion of the Preisach hysteresis operator with application to control of smart actuators," IEEE Trans. Automat. Contr., vol. 50, pp. 798-810, 2005.

[10] C.J. Lin, S.R. Yang, "Precise positioning of piezo-actuated stages using hysteresisobserver based control," Mechatronics, vol. 16, pp. 417-426, 2006.

[11] H. Logemann, A.D. Mawby, "Low-gain integral control of infinite-dimensional regular linear systems subject to input hysteresis," Advances in Mathematical Systems Theory (edited by F. Colonius et al), pp. 255-293, Birkhäuser, Boston, 2001.

[12] H. Logemann, E.P. Ryan, "Systems with hysteresis in the feedback loop: Existence, regularity and asymptotic behaviour of solutions," ESAIM: Control, Optimization and Calculus of Variations, vol. 9, pp. 169-196, 2003.

[13] H. Logemann, E.P. Ryan, I. Shvartsman, "Integral control of infinite-dimensional systems in the presence of hysteresis: an input-output approach," ESAIM: Control, Optimization and Calculus of Variations, vol. 13, pp. 458-483, 2007.

[14] I. Mayergoyz, Mathematical Models of Hysteresis and Their Applications, Academic Press, Boston, 2003.

[15] P.D. Mitchler, R.M. Roshko, E. Dan Dahlberg, E. Wesseling, "Preisach model for spin-glass remanences," Physical Review B, vol. 55, pp. 5880-5885, 1997. 
[16] Y.W. Park, M.C. Lim, D.Y. Kim, "Control of a magnetostrictive actuator with feedforwarding inverted hysteresis," Journal of Magnetism and Magnetic Materials, vol. 272-276, Supplement 1, pp. E1757-E1759, 2004.

[17] G. Song, J. Zhao, X. Zhou, J.A. De Abreu-García, "Tracking control of a piezoceramic actuator with hysteresis compensation using inverse Preisach model," IEEE/ASME Trans. Mechatronics, vol. 10, pp. 198-209, 2005.

[18] W. Symens, F. Al-Bender, J. Swevers, H. van Brussel, "Dynamic characterization of hysteresis elements in mechanical systems," Proc. IEEE American Control Conf., pp. 4129-4134, Anchorage, 2002.

[19] X. Tan, J.S. Baras, P.S. Krishnaprasad, "Control of hysteresis in smart actuators with application to micro-positioning," Systems $\&$ Control Letters, vol. 54, pp. 483-492, 2005.

[20] Y.K. Wen, "Method of random vibration of hysteretic systems", J. Eng. Mech. ASCE, vol. 102, pp. 249-263, 1976. 